\title{
Developing Tools for STEM Education: The Foldscope, a Very Inexpensive Monocular Microscope for Biological Research.
}

\author{
Frank Denaro $^{1}$, Maya Gabriel ${ }^{1}$, Arsene Noe $^{1}$, and Simon Nyaga ${ }^{1}$ \\ 1. Department of Biology, Morgan State University, Baltimore, MD, USA
}

The microscope, you will no doubt point out, is an expensive piece of equipment. So much so, that it is impossible to lend or give one to each student for personal use. Departmental scientific teaching budgets continue to be problematic. Even through grants are submitted, often times they are not funded, which can mean a restriction in the student's STEM experience [1]. Recent developments are changing the approach to this situation. One idea that is now gaining a following is that of "frugal science". Although this concept was stressed about hundred years ago in the autobiographical writings of the Nobel Laureate Dr. Santiago Ramón y Cajal, it has been ignored until fairly recently. His essential view was to begin research and scientific thinking with what is available to you. ("The person is everything, the means are but little.", S. Ramon y Cajal). A view that was as radical then, as it is today. But today we have a vast array of inexpensive (and high quality) scientific tools, that were not available in Cajal's time. An extremely important research tool that is now affordable is the microscope, in particular the monocular microscope. By adapting the historic monocular microscope (like the one Anton van Leeuwenhoek made 1632 to 1723 !) with new materials and technology (such as plastics, paper, light emitting diodes, and the cell phone camera) it is possible to produce very inexpensive microscopes which overcome some of the financial barriers confronted with this particular tool. These scopes are capable of $\sim 140 \mathrm{x}$ magnification with $2 \mathrm{mu}$ resolution [2]. This makes them very useful microscopes because the cell can be observed. They are now commercially available and at prices which can allow each student to have a microscope. In the present study we have used a commercially available monocular scope, the Foldscope (https://www.foldscope.com/) and explored its capabilities as a research instrument.

The pros and cons of the monocular scope are interesting. Because it is monocular, the multiplying effects of a multi-lens system that increases chromatic and spherical aberration are minimized. In the compound microscope, one needs special correction of the lens to minimize these problems. This increases expensive. Such corrections are not needed in monocular scopes because the spherical and chromatic aberrations are minimal with one lens. The working distance is very close and the eye must be put up right near the lens. This is an ergonometric problem when intense slide examination is required. But now with very good cell phone cameras, direct imaging can go via the cell phone camera. Alternatively, a frosted sheet (thin velum) can be placed above the lens and the image can be viewed on it. The body of the Foldscope is paper. This lowers the price, but inhibits the exploration of the slide, because movement of the slide and/or focus adjustment, can result in the need to realign the image/slide. The creation of a platform to stabilize the scope helps with this problem and allows the slide to be systematical observed with a bit less realignment. We have made a simple support from a piece of packing Styrofoam. High quality cover slips can also improve the image. Without the ability to switch between low and high power, slide investigation is difficult. Enumerated gradations on the coverslips would help the investigator to know which parts of the slide they are observing. This is important for diagnostics. An adaption of the scope for the observation of continuous cell cultures would be helpful. Also a more adaptable stage that permits better slide scanning would be a big help. We are examining modifications for these possibilities. Image analysis is now an important part of microscopy. The cell phone camera can do a lot of post image capture processing to magnify and improve the image. If a cell phone "APP" was developed to do some image processing like pixel intensity, cell counts and data processing, the scope would have greater research potential. 
Construction out of a material that can be sterilized would be very helpful. Also resistance to cover slipping media would be a plus.

We have observed some H\&E stained sections of tissue, cell culture preps, and immunohistochemical labels. Staining makes for ease of location, unstained cell cultures are a bit harder to locate and image but it is possible. Review of large slide numbers is a bit tedious and that makes slide review for research projects a commitment. But, for teaching purposes, the students do not have that difficulty [3].

\section{References:}

[1] Frank Denaro and Simon Nyaga, A microscope of your own: Introduction of the personnel microscope to STEM Educational programs and research. The 2018 Capital PKAL Regional Network Conference Cultural Responsiveness in Fulfilling the Mission of Preparing a Global STEM Workforce, March 16, 2018, Stevenson University, Owings Mill, Maryland

[2] Cybulski JS, Clements J, Prakash M, Foldscope: Origami-Based Paper Microscope. PLoS ONE 9(6) (2014) p. e98781. https://doi.org/10.1371/journal.pone.0098781

[3] We acknowledge ASCEND Grant: 5 UL1 GM118973, and R29 NS31857-01A1.
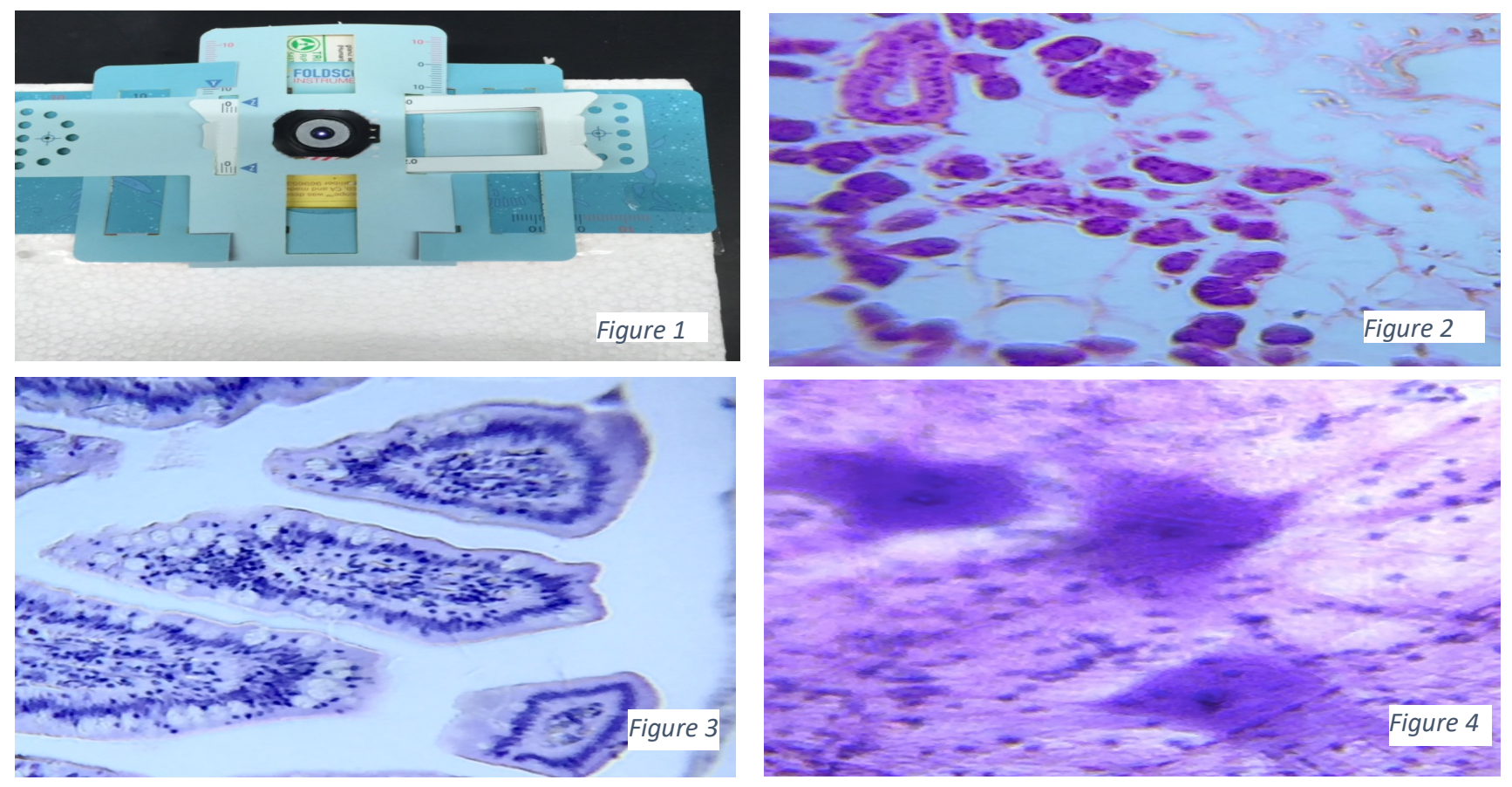

Figures 1-4. Foldscope pictures taken by cell phone. In the Fig. 1 we have sunk the light source into a Styrofoam support and taped part of the scope to it. This creates improved stability for slide observation. The cell phone can then be placed on top and the slide observed. Fig. 2, Salivary gland, Fig. 3 Intestine, and Fig. 4 Neural cells, are all examples of H\&E stained tissue recorded by cell phone. The image quality is reasonable, it is possible to observe cells and nuclei. Experimental alterations to cells, cell counts, and special stains can also be observed. While the Foldscope has a "slide stage", adaptations to it may make it more convent for the study of multiple slides. This is a necessity for research projects where many slides must be viewed. 